

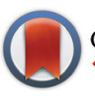

CrossMark

\&lick for updates

Cite this: Polym. Chem., 2017, 8, 674

\section{Synthesis of polycaprolactone-polyimide- polycaprolactone triblock copolymers via a 2-step sequential copolymerization and their application as carbon nanotube dispersants $\uparrow$}

\begin{abstract}
Chengyin Liu,ța Bo Liuț and Mary B. Chan-Park*a,b
Polyimides have attractive properties such as a strong interaction with 1D/2D carbon nanomaterials but their solubilities in common organic solvents are limited. We report a new synthesis route for triblock copolymers of polycaprolactone-polyimide-polycaprolactone (PCL-PI-PCL) via polycondensation followed by ring-opening polymerization. The prepared $\mathrm{OH}-\mathrm{PI}-\mathrm{OH}$ homopolymer precursors were reacted with two equivalents of stannous(II) octoate $\left(\mathrm{Sn}(\mathrm{Oct})_{2}\right)$ to afford $\alpha, \omega$-dihydroxyl-terminated polyimide macroinitiators which can polymerize with $\varepsilon$-caprolactone to obtain the final triblock copolymers (PCL-PI-PCL). Four different molecular weights of PCL-PI-PCL triblock copolymers with different lengths of PCL and PI blocks were synthesized to assess the best composition for carbon nanotube dispersion in a low boiling organic solvent (tetrahydrofuran, THF). The polyimide block interacts strongly with single walled carbon nanotubes (SWNTs) through charge transfer, as shown by Raman spectroscopy, while the polycaprolactone block has a good solubility in THF. An optimised triblock copolymer disperses the carbon nanotubes in THF well even after standing for $1 \mathrm{~h}$, while the PI homopolymer-dispersed SWNTs settled completely under the same conditions. We applied the new PCL-PI-PCL in SWNT-reinforced epoxy composites with the use of THF as the casting solvent. The optimised triblock copolymerdispersed SWNTs (2 wt\%) increased the tensile strength, modulus, and elongation at maximum stress by $74 \%, 35 \%$, and $62 \%$ respectively compared to the neat resin blend. The new synthesis route of the triblock copolymer is amenable to the synthesis of diverse PI-based triblock copolymers with various desired functionalities for myriad applications, such as for carbon nanotube-reinforced epoxy-based composites, water-based antibacterial dispersions, etc.
\end{abstract}

Received 6th November 2016 Accepted 12th December 2016 DOI: $10.1039 /$ c6py01933a www.rsc.org/polymers

\section{Introduction}

Polyimides are high-performance aromatic materials with interesting properties such as high glass transition temperatures and strong interaction with $1 \mathrm{D} / 2 \mathrm{D}$ carbon nanomaterials. ${ }^{1}$ We had previously shown that polyimides (PIs) were very good dispersing agents for carbon nanotubes because of their strong $\pi-\pi$ interaction with carbon nanotube side walls. ${ }^{2,3}$ However, they were only soluble in polar aprotic solvents such as DMSO, NMP and DMF. Their poor solubilities

\footnotetext{
${ }^{a}$ School of Chemical and Biomedical Engineering, Nanyang Technological University, 62 Nanyang Drive, Singapore 637459, Singapore. E-mail: MBECHAN@ntu.edu.sg ${ }^{b}$ Lee Kong Chian School of Medicine, Nanyang Technological University, Singapore $\dagger$ Electronic supplementary information (ESI) available: Fig. S1-S14 and Tables S1-S3. See DOI: 10.1039/c6py01933a

\$These two authors contributed equally.
}

and incompatibility with common organic solvents such as THF, chloroform and acetone limited their applications. It would be desirable to modify PI in order to make it more soluble in common solvents. The sequential copolymerization of block copolymers of polyester-polyimide-polyester (PE-PI-PE) has not been reported. Ju et al. synthesized a triblock polycaprolactone-block-polyimide-block-polycaprolactone (PCL-PI-PCL), for use as a dielectric material, via "grafting to" of PCL-furyl amine to maleimide-PI. ${ }^{4}$ However, the "grafting to" method may be somewhat limited by the efficiency and solvent compatibility of the two blocks. They linked PCL and PI by the Diels-Alder reaction, which was reversible, so the triblock copolymer may decompose with heating. Other polyimide-vinyl block copolymers have been synthesized via atom transfer radical polymerization (ATRP), including polyimidegraft-polystyrene and poly(methyl methacrylate)-block-polyimide-block-poly(methyl methacrylate). ${ }^{5-7}$ Polyimide-polyester 
block copolymers have hitherto not been directly synthesized. Ring-opening polymerization has not previously been employed in the synthesis of PI-based copolymers.

Single walled carbon nanotubes (SWNTs) are high-performance nanomaterials being exploited for diverse applications such as polymer matrix composites, antibacterial nanomaterials ${ }^{8}$ field-effect transistors,${ }^{9,10}$ biosensors, ${ }^{11,12}$ etc. Their intrinsic mechanical, electrical and optical properties are outstanding. ${ }^{13}$ However, realization of these properties in practice typically depends on good dispersion of SWNTs in common solvents such as THF, dichloromethane (DCM), acetonitrile, etc. The high surface area of nanotubes results in strong intertube van der Waals forces ${ }^{14}$ so that they easily aggregate into bundles. Nanotube dispersing agents ought to have strong affinity for the nanotubes as well as good solubilities in common solvents, which may entail contrasting compositional requirements for dispersing agents. Both covalent and noncovalent functionalization approaches have been reported. ${ }^{14-16}$ Covalent functionalizations can improve dispersion but typically destroy the long-range $\pi$ conjugation of SWNTs. ${ }^{17}$ Noncovalent functionalizations are usually realized through physical adsorption, which does not disrupt the intrinsic structure of SWNTs, but the types of polymers and organic solvents that can be applied are still limited. ${ }^{18,19}$ Recently, conjugated and aromatic polymers/copolymers have attracted much attention as dispersing agents. ${ }^{20-22}$

In this paper, we prepared three polyimide homopolymers with different molecular weights (Table 1) and used them as centre blocks for synthesizing a series of polycaprolactoneblock-polyimide-block-polycaprolactone (PCL-PI-PCL) triblock copolymers via a novel synthesis route (Scheme 1). ABA block copolymers were synthesized through addition of hydroxyl $(-\mathrm{OH})$ active groups to the two ends of the centre PI blocks, followed by ring-opening polymerization of the PCL end blocks (Scheme 1). ${ }^{7,23}$ Three PI homopolymers with different molecular weights (labelled as HP1, HP2 and HP3, Table 1)

Table 1 Synthesis of $\mathrm{OH}$-bifunctional polyimides

\begin{tabular}{llrl}
\hline Sample & Molar ratio in feed $[\mathrm{BPADA}] /[\mathrm{HAB}] /[\mathrm{APA}]$ & $M_{\mathrm{n}}{ }^{a}(\mathrm{Da})$ & $\mathrm{PDI}^{a}$ \\
\hline HP1 & $100 / 75 / 50$ & 6000 & 1.78 \\
HP2 & $100 / 90 / 20$ & 14700 & 1.65 \\
HP3 & $100 / 95 / 10$ & 35800 & 1.80
\end{tabular}

${ }^{a}$ Determined from GPC using polystyrene standards as references.

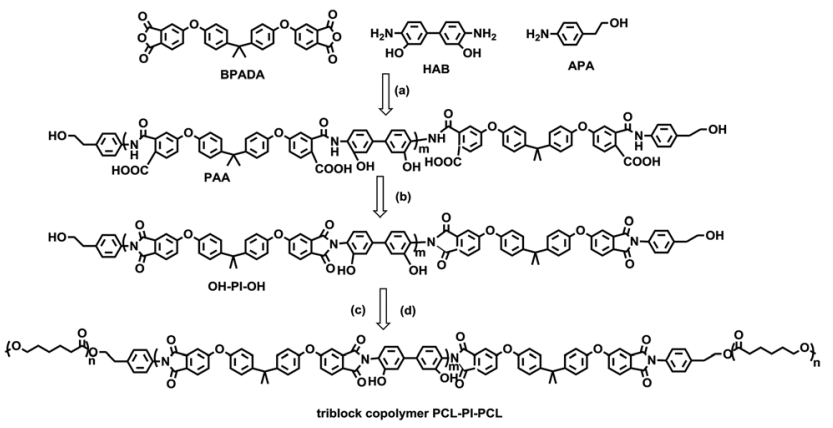

Scheme 1 Synthesis of the PCL-PI-PCL triblock copolymer: (a) dry NMP, $25^{\circ} \mathrm{C}, 10 \mathrm{~h}$; (b) dry $p$-xylene, reflux, $6 \mathrm{~h}$; (c) Sn(Oct) $)_{2}$ dry NMP, $25^{\circ} \mathrm{C}, 6 \mathrm{~h}$ (d) $\mathrm{CL}, 100{ }^{\circ} \mathrm{C}, 24 \mathrm{~h}$.

and four triblock copolymers with these PI centre blocks were synthesized (labelled as TB1a, TB1b, TB2 and TB3, see Table 2). The (co)polymers were characterized with NMR, GPC and FTIR. SWNT dispersions, using PCL-PI-PCL triblock copolymers (TBs) and homopolyimide (HP1), were characterized with UV-VIS-NIR, AFM, TEM, and Raman spectroscopy. We found that the TB1a triblock copolymer most effectively dispersed SWNTs. The central PI block noncovalently adsorbed on carbon nanotubes while the two ends of PCL blocks provided good solubilities in THF solvent (a model low boiling point solvent). There appeared to be an optimum ratio of PI to PCL block length for the triblock (as with TB1a) to achieve excellent SWNT dispersion in THF solvent. Furthermore, epoxy composites reinforced with SWNTs dispersed by TB1a in THF were fabricated and the tensile properties were improved by incorporation of the SWNTs.

\section{Results and discussion}

\section{Design and synthesis of $\mathrm{OH}-\mathrm{PI}-\mathrm{OH}$ homopolymers and PCL-PI-PCL copolymers}

The synthesis route of OH-PI-OH homopolymers and PCL-PI-PCL triblock copolymers is shown in Scheme 1. The $\mathrm{OH}-\mathrm{PI}-\mathrm{OH}$ homopolymers were synthesized via a two-step reaction starting from BPADA, HAB and APA dissolved in NMP. During the first step, the three monomers underwent polycondensation at room temperature to form a yellow color viscous poly(amic acid) solution, while in the second step at

Table 2 PCL-PI-PCL triblock copolymers initiated by different polyimide homopolymers in Table 1

\begin{tabular}{|c|c|c|c|c|c|c|}
\hline TB1a & $1: 100$ & HP1 (6000) & 13 200/1.40 & 9 & 60 & $3: 1: 3$ \\
\hline TB2 & $1: 200$ & HP2 (14 700) & 17 700/1.43 & 21 & 24 & $1: 2: 1$ \\
\hline TB3 & $1: 200$ & HP3 (35 800) & $39300 / 1.65$ & 51 & 28 & $3: 10: 3$ \\
\hline
\end{tabular}

${ }^{a}$ Molar ratio of polyimide macroinitiator and caprolactone monomers for ring-opening polymerization. ${ }^{b}$ Determined from GPC using polystyrene standards as references. ${ }^{c}$ Calculated from the molecular weight of polymers and single units. 
$180{ }^{\circ} \mathrm{C}$, a yellowish polyimide was formed after thermal imidization. Two terminal $\mathrm{OH}$ groups of polyimide are observed in Fig. $\mathrm{S} 1(\mathrm{~b}) \dagger\left(\delta_{-\mathrm{OH}}=4.76 \mathrm{ppm}, \delta_{-\mathrm{CH}_{2}-\mathrm{OH}}=3.50 \mathrm{ppm}\right.$ and $\delta_{-\mathrm{CH}_{2}-\mathrm{CH}_{2}}=$ $2.77 \mathrm{ppm})$. The measured molecular weights $\left(M_{\mathrm{n}}\right)$ of various $\mathrm{OH}-\mathrm{PI}-\mathrm{OH}$ increased from $6000 \mathrm{Da}$ to $14700 \mathrm{Da}$ and to $35800 \mathrm{Da}$ with the increasing ratio of $\mathrm{HAB}$ monomers; three polyimide homopolymers, labelled as HP1 to HP3 (Table 1), were synthesized.

The as-prepared OH-PI-OH (HP1 to HP3) can easily react with $\mathrm{Sn}(\mathrm{Oct})_{2}$ to yield an $\alpha, \omega$-OSn(Oct) terminated PI initiator, which can undergo ring-opening polymerization with cyclic ester monomers, such as $\varepsilon$-caprolactone, lactones, carbonates, etc. We prepared four PCL-PI-PCL triblock copolymers, labelled as TB1a, TB1b, TB2 and TB3, from the HP1 to HP3 center blocks (Table 2). The four triblock copolymers were characterized by NMR and GPC. For example, successful synthesis of the triblock copolymer TB1a was confirmed with ${ }^{1} \mathrm{H}$ NMR and FTIR. The chemical shifts at 4.00, 2.30, 1.56 and $1.32 \mathrm{ppm}$, which were absent in homopolymer spectra, were assigned to the PCL block (Fig. S1 $\dagger$ ). The FTIR spectrum of the triblock copolymer had bands around 2800-3000 $\mathrm{cm}^{-1}(\mathrm{C}-\mathrm{H}$ stretching) which can be assigned to the characteristic absorption bands of PCL components. Furthermore, the characteristic bands of polyimides around $1730 \mathrm{~cm}^{-1}(\mathrm{C}=\mathrm{O}$ stretching), $1360 \mathrm{~cm}^{-1}$ (C-N stretching) and $1200 \mathrm{~cm}^{-1}$ (aromatic-OH stretching) were observed in both HP1 and TB1a (Fig. S2 $\dagger$ ). The syntheses of the other PI homopolymers (HP2 and HP3) and PCL-PI-PCL triblock copolymers (TB1b, TB2, and TB3) were also confirmed by ${ }^{1} \mathrm{H}$ NMR (Fig. S3 to S7 $\dagger$ ).

Fig. S11† shows the GPC curves of OH-PI-OH homopolymers (HP1, HP2, and HP3) and also the PCL-PI-PCL triblock copolymers (TB1a, TB1b, TB2, and TB3). The molecular weights $\left(M_{\mathrm{n}}\right)$ of PI and PCL-PI-PCL are summarized in Tables 1 and 2, respectively. The molecular weights of all the triblock copolymers exceeded those of the corresponding $\mathrm{OH}-\mathrm{PI}-\mathrm{OH}$ polymers. For example, $M_{\mathrm{n}}$ of the homopolymer HP1 was $6000 \mathrm{Da}$, but that of TB1a (the triblock made from HP1) was 13200 Da. The PDI of the triblock remained narrow (1.2 to 1.8, Table 2), indicating that the PCL-PI-PCL was a true triblock copolymer and not a mixture.

The solubilities of these polymers in common solvents are shown in Tables S1 and S2. $\dagger$ TB1a and TB1b were soluble in more solvents because they had short polyimide center blocks; TB3 was soluble in only a few solvents because of the high molecular weight of its polyimide block.

\section{SWNT dispersion and interfacial bonding with PCL-PI-PCL}

SWNT dispersions in THF using various PCL-PI-PCL triblock copolymers as dispersing agents were quantitatively evaluated using UV-VIS-NIR absorption spectra and the Beer-Lambert law (see Experimental details). The absorbance of dilute polymer TB1a solutions $(0.025,0.05,0.075,0.125$ and 0.2 $\mathrm{mg} \mathrm{mL} \mathrm{m}^{-1}$ ) with SWNTs (mass ratio of TB1a and SWNTs $=2: 1$ ) in THF was measured and the absorbance at $700 \mathrm{~nm}$ was plotted against SWNT concentrations (Fig. 1). A linear-leastsquares fit to the data gave a slope of 11.7 , which was the

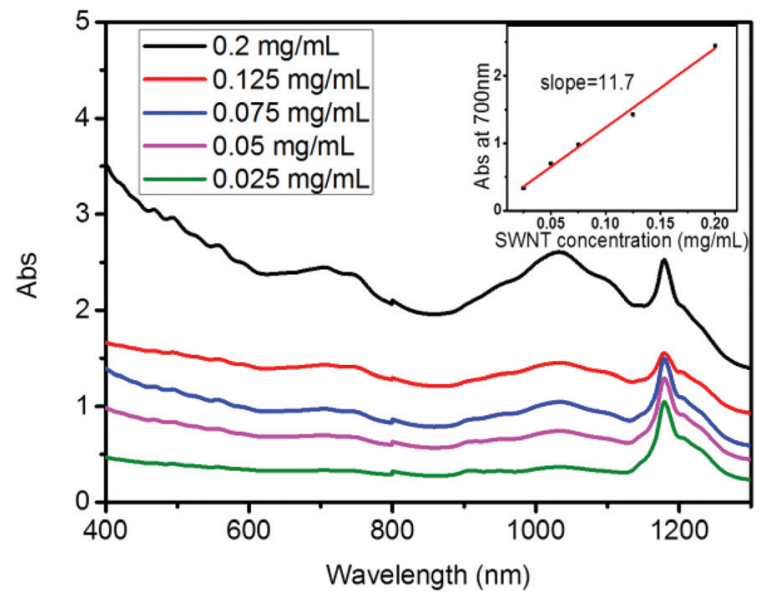

Fig. 1 Absorption spectra of SWNTs with TB1a dispersant (mass ratio of TB1a and SWNTs = 2:1) at different nanotube concentrations immediately after nanotube suspension. Inset shows the concentration dependence of the absorbance at $700 \mathrm{~nm}$. The straight line is a linear-leastsquares fit to the data.

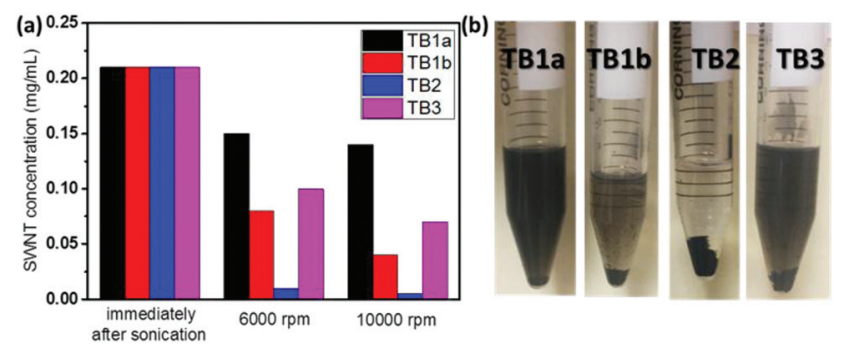

Fig. 2 (a) SWNT concentrations of different molecular weight PCL-PI-PCL dispersed in THF under different conditions: immediately after sonication, after centrifugation at $6000 \mathrm{rpm}$ for $10 \mathrm{~min}$, and after centrifugation at $10000 \mathrm{rpm}$ for $10 \mathrm{~min}$; (b) photographs of PCL-PI-PCL dispersed SWNTs in THF after centrifugation at 10000 rpm.

extinction coefficient (i.e., $\varepsilon=11.7 \mathrm{~mL} \mathrm{mg}^{-1} \mathrm{~cm}^{-1}$ ) for back-calculating the SWNT concentration in other solutions (the dispersing agent and solvent absorbance are low and smoothly varying at the wavelength of our measurements, and so can be easily subtracted from the absorbance measurements of dispersed SWNTs) (Fig. S13†).

We evaluated the different TBs (TB1a, TB1b, TB2 and TB3) for dispersing SWNTs in THF (Fig. 2). The nanotube concentrations of the supernatants of various TB/SWNT dispersions subjected to various settling regimes, i.e. specifically immediately after sonication, or sonication followed by centrifugation at $6000 \mathrm{rpm}$ or $10000 \mathrm{rpm}$ each for $10 \mathrm{~min}$, were evaluated by UV-VIS-NIR and the Beer-Lambert law. For all the TBs, some SWNTs settled at the bottom after centrifugation at $6000 \mathrm{rpm}$ or $10000 \mathrm{rpm}$, while some still remained in the solvent (Fig. 2). After centrifugation at the higher speed (10000 rpm), TB1a-dispersed SWNT solution had the highest nanotube concentration (Fig. 2(a)), indicating that TB1a was the most effective in dispersing SWNTs. TB2-dispersed SWNT solution was almost clear and the color of TB1b-dispersed SWNT 
solution was light after centrifugation (Fig. 2(b)), indicating that most SWNTs have settled at the bottom. Comparing the four TB dispersants, the length of PCL should be moderate; too long (TB1b) or too short PCL block (TB2) impaired the dispersion; a longer PI block while keeping the PCL block length constant was helpful for the nanotube dispersion, which was why TB3 was better than TB2 in dispersing the nanotubes. In order to confirm this statement, more TBs with different lengths of PCL blocks were made (TB1c, TB1d and TB1e, see Table S3†). They were all initiated by the same polyimide (HP1) and their molecular weights are summarized together with TB1a and TB1b in Table S3. $\dagger$ The synthesis of TB1c, TB1d and TB1e was confirmed by ${ }^{1} \mathrm{H}$ NMR spectra (Fig. S8-S10†) and GPC curves (Fig. S12†). The molecular weights of the newly synthesized TB1C and TB1d are 9400 and 11500 daltons, whereas the TB1e molecular weight is 16600 daltons, an intermediate between TB1a and TB1b. The concentrations of SWNTs in THF dispersed by different TBs are calculated by using UV-Vis-NIR curves and are shown in Fig. S14. $\dagger$ Immediately after sonication, the concentrations of SWNTs are all around $0.2 \mathrm{mg} \mathrm{mL}^{-1}$, while after centrifugation at $6000 \mathrm{rpm}$ and 10000 rpm, the concentration of SWNTs using TB1a and TB1e dropped to the least compared to the other 3 ratios, indicating that a better dispersant is achieved by optimizing the ratio of PI to PCL block chain lengths to be around $1: 3$. Considering these various factors (different PCL block length ratios and PI lengths), TB1a was among the best TBs to effectively disperse SWNTs in THF. So we choose TB1a as a dispersant to further evaluate the dispersion of SWNTs and make some composites.

We also compared the efficacy of the polyimide homopolymer (HP1) center block versus the TB1a triblock copolymer in suspending SWNTs in THF after centrifugation, by comparing the SWNT content in solutions of HP1/SWNT versus that of TB1a/SWNT (Fig. 3(a)). Immediately after sonication, both TB1a/SWNT and HP1/SWNT dispersion had nearly the same nanotube concentration $\sim 50 \mathrm{mg} \mathrm{L}^{-1}$. After centrifugation, the nanotube concentrations in the supernatants of TB1a/SWNT decreased to 33 and $28 \mathrm{mg} \mathrm{L}^{-1}$ for $6000 \mathrm{rpm}$ and $10000 \mathrm{rpm}$ respectively, which were much higher than the corresponding values of HP1/SWNT ( 6 and $3 \mathrm{mg} \mathrm{L}^{-1}$ ) after similar centrifugation regimes (Fig. 3(a)), indicating that the dispersion of SWNTs in THF was greatly improved by incorporation of PCL end blocks in the copolymers. We also compared the settling

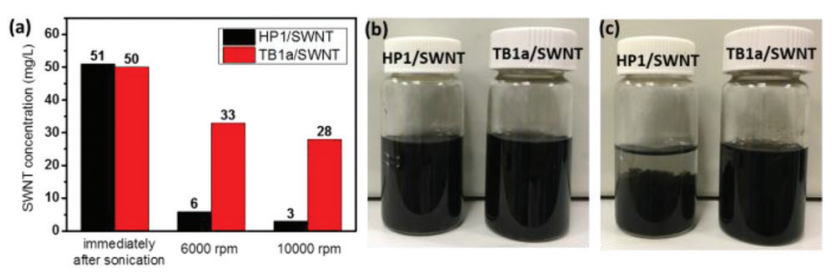

Fig. 3 SWNT concentrations of HP1 dispersed and TB1a dispersed in THF under different conditions: (a) immediately after sonication, after centrifugation at $6000 \mathrm{rpm}$ for $10 \mathrm{~min}$, and after centrifugation at $10000 \mathrm{rpm}$ for $10 \mathrm{~min}$. Photographs of SWNTs dispersed in THF after sonication immediately (b) and after standing for $1 \mathrm{~h}$ (c). of SWNTs upon standing after $1 \mathrm{~h}$ using HP1 (control) versus TB1a as dispersing agents; Fig. 3(b) and (c) show the photographs of SWNTs dispersed in THF after standing for $1 \mathrm{~h}$ and immediately after sonication respectively. After sonication, both the solutions were black (Fig. 3(b)). But after standing for $1 \mathrm{~h}$, the SWNTs dispersed in HP1 solution mostly settled, while no obvious aggregates were observed in the TB1a/SWNT solution, indicating that homogeneous dispersion that was stable on standing was achieved with the TB1a triblock polymer (Fig. 3(c)). Because the solubility of PCL in THF was very good (Table $\mathrm{S} 2 \uparrow$ ), it can improve the solubility of SWNTs in the solvent and help to form a more stable SWNT solution.

To further examine the nanotube dispersion and morphology, TEM and AFM images of the dispersed SWNTs were obtained. The HP1-dispersed SWNTs, which were used as the control, formed bundles with large diameters of about $30 \mathrm{~nm}$ (Fig. 4(a)), while the majority of the TB1a-dispersed SWNTs was observed in smaller bundles with diameters of around $5 \mathrm{~nm}$ (Fig. 4(b)i). At a lower magnification $(\times 8000)$ (Fig. 4(b)ii), it can be seen that the carbon nanotubes were wrapped with the PCL-PI-PCL polymer (TB1a) and also intercalated by the polymers, to which the superior dispersion and long-term stability of the SWNT solution may be attributed. The ability of the triblock copolymer to disperse SWNTs into smaller bundles and individual tubes was also manifested by AFM (Fig. 5). The measured height of the SWNTs was below $5 \mathrm{~nm}$, and some were 1-2 nm, suggesting that the SWNTs were dispersed as small bundles and individual tubes.

To confirm the strong interaction between the synthesized polymer and carbon nanotubes, $785 \mathrm{~nm}$ laser Raman spectra of pristine SWNTs and different polymer (PCL, HP1 and TB1a) dispersed SWNTs were obtained (Fig. 6). It was known that covalent functionalization can introduce defects into SWNTs, leading to an increased intensity of the D band $\left(\sim 1330 \mathrm{~cm}^{-1}\right) \cdot{ }^{24,25}$ On non-covalent functionalization with the various polymers (PCL, HP1 and TB1a), the intensity ratios of the $\mathrm{D}$ band to the $\mathrm{G}$ band $\left(I_{\mathrm{D}} / I_{\mathrm{G}}\right)$ of the various dispersions were not larger than that of unfunctionalized SWNTs (Fig. 6(a)), corroborating that the nanotube graphene structure was well preserved. However, the $\mathrm{G}+$ band showed varying degrees of shift. For all three polymers treated with SWNTs, the $\mathrm{G}+$ band shifted from $1592.5 \mathrm{~cm}^{-1}$ to $1589.6 \mathrm{~cm}^{-1}$, indicating n-doping due to charge transfer (Fig. 6(a)). ${ }^{26}$ The electrondonating behaviour of PCL was due to the oxygen and alkyl
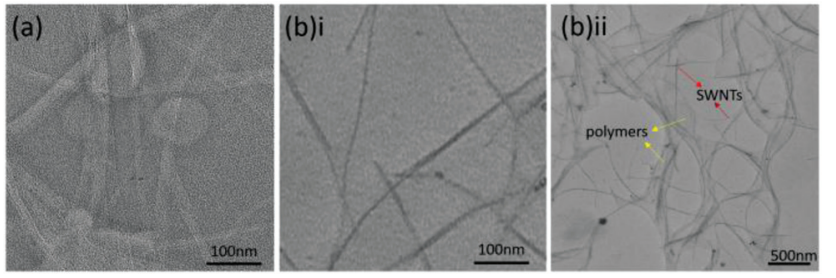

Fig. 4 TEM images of dispersed SWNTs with (a) HP1 (control) ( $\times 50000$ magnification); (b) (i) TB1a ( $\times 50000$ magnification); (b) (ii) TB1a ( $\times 8000$ magnification). 

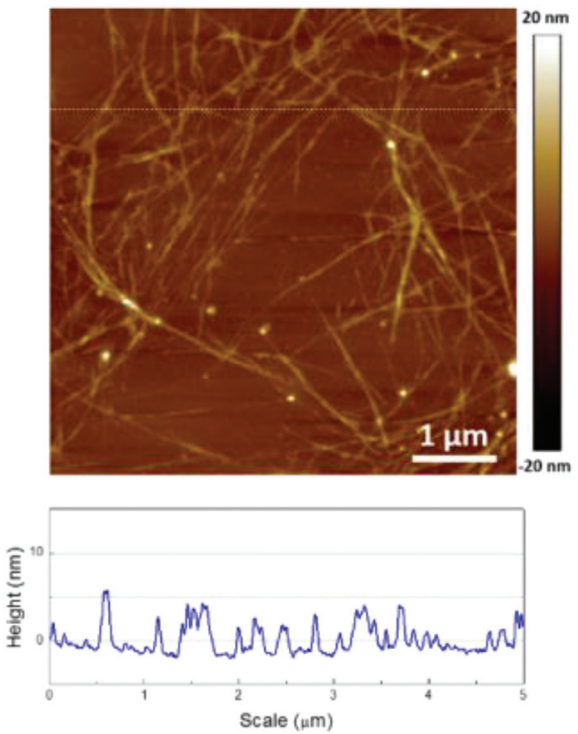

Fig. 5 AFM image of TB1a-dispersed SWNTs deposited on silicon wafer by drop-casting dilute solution $\left(0.01 \mathrm{mg} \mathrm{mL}^{-1}\right)$.
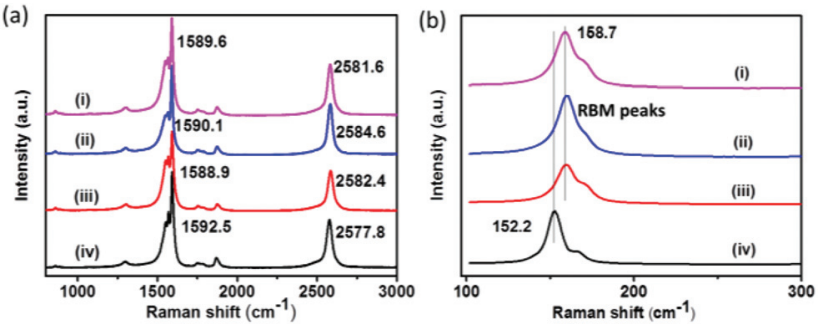

Fig. 6 Raman spectra (785 nm laser) of polymer-dispersed SWNTs: (i) TB1a/SWNT; (ii) PCL/SWNT; (iii) HP1/SWNT and (iv) pristine SWNTs $\left(I_{\mathrm{D}} / I_{\mathrm{G}}=\right.$ (i) 0.122 ; (ii) 0.127 ; (iii) 0.117 ; (iv) 0.173 ).

chain on it while that from PI was due to the electrons of the aromatic rings. The G+ peak of HP1/SWNT, PCL/SWNT and TB1a/SWNT shifted by $3.6 \mathrm{~cm}^{-1}, 2.5 \mathrm{~cm}^{-1}$, and $2.9 \mathrm{~cm}^{-1}$ respectively so that the shift of TB1a/SWNT was intermediate between HP1/SWNT and PCL/SWNT. From the Raman shift of HP1 and TB1a, the polyimide (either by itself or in the triblock copolymers) had a strong interaction with the nanotubes and was an effective way to increase the interaction between the polymer dispersants and carbon nanotubes. The Raman radial breathing mode (RBM) peaks also became broader due to the interaction between the polymer and SWNTs, ${ }^{27}$ and the change of the shape was more obvious in HP- and TB-dispersed carbon nanotubes (Fig. 6(b)i and iii) (the RBM frequency was related to the radius of carbon nanotubes, so the up-shifts of RMB peaks were attributed to the polymer wrapping ${ }^{28}$ ).

\section{SWNT dispersion in TB1a/epoxy films and the mechanical properties of the composite films}

As TB1a was the best SWNT dispersant of the four TBs synthesized, we used it as the dispersant of nanotubes for epoxy

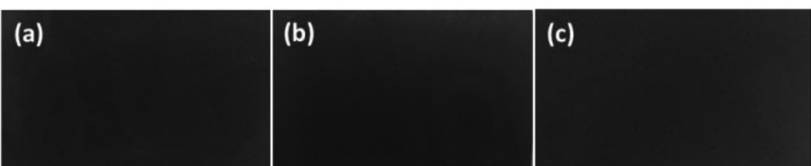

Fig. 7 Visual appearance of the TB1a/epoxy/SWNT composite film (a) 2 wt\% SWNTs, (b) 4 wt\% SWNTs, and (c) 6 wt\% SWNTs.

composite fabrication with a range of SWNT loadings. Some typical TB1a/epoxy/SWNT composite films of different loadings are shown in Fig. 7. The appearance of films is quite dark and homogeneous for all three loadings ( $2 \mathrm{wt} \%, 4 \mathrm{wt} \%$ and $6 \mathrm{wt} \%)$. The mechanical properties of the films are summarized in Table 3. Typical stress-strain curves of the composite films are shown in Fig. 8. Among all the composite films, $2 \mathrm{wt} \%$ SWNT loading achieved the best mechanical properties. Its strength, modulus and elongation at maximum stress are $74 \%$, $35 \%$ and $62 \%$, respectively, higher than those of the TB1a/epoxy control film made with 0 wt $\%$ SWNTs. The modulus of the composite films increased when increasing the amount of carbon nanotubes. At the higher SWNT loading, the strength and elongation properties decreased. This may be due to the increase of free volume in the composite films brought by SWNTs. $^{29}$

Fracture surfaces of the composite films after tensile testing are shown in Fig. 9. There were some pull-outs observed for the $2 \%$ SWNT fractured surface but they were not too exten-

Table 3 Mechanical properties of TB1a/SWNT/epoxy composite films

\begin{tabular}{llll}
\hline $\begin{array}{l}\text { SWNT } \\
\text { loading } \\
(\text { wt\%) }\end{array}$ & $\begin{array}{l}\text { Strength }(\mathrm{MPa}) \\
(\text { increase\%) }\end{array}$ & $\begin{array}{l}\text { Modulus }(\mathrm{MPa}) \\
\text { increase\%) }\end{array}$ & $\begin{array}{l}\text { Elongation } \\
\text { at maximum } \\
\text { stress }(\%)\end{array}$ \\
\hline 0 & $35.3 \pm 6.6$ & $1709.9 \pm 191.7$ & $3.40 \pm 1.16$ \\
2 & $61.4 \pm 2.2(74)$ & $2307.0 \pm 136.3(35)$ & $5.50 \pm 1.04$ \\
4 & $48.5 \pm 3.2(37)$ & $2318.5 \pm 184.7(36)$ & $4.24 \pm 0.81$ \\
6 & $45.2 \pm 4.3(28)$ & $2431.1 \pm 107.6(42)$ & $3.46 \pm 0.48$ \\
\hline
\end{tabular}

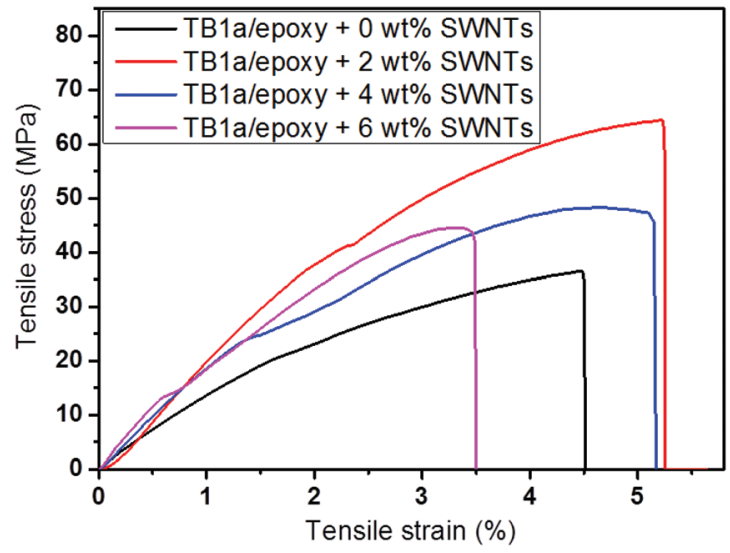

Fig. 8 Typical stress-strain curves of TB1a/epoxy/SWNT composite films. 


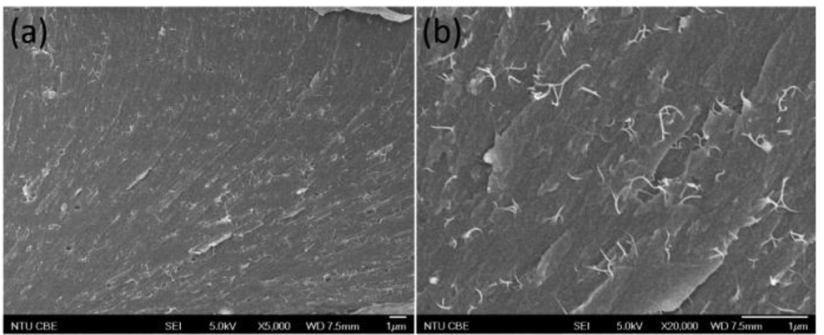

Fig. 9 FE-SEM images of the fracture surface of TB1a/epoxy/SWNT with 2 wt\% SWNT loading at (a) low magnification $(\times 5000)$ and (b) high magnification $(\times 20000)$.

sive. The SWNTs were well dispersed in the composite films and no obvious agglomeration was observed, indicating that the PCL-PI-PCL triblock (TB1a) was an effective SWNT dispersant for epoxy composites.

\section{Experimental}

\section{Materials}

P2-SWNTs (purity $=90 \%$, diameter $4-5 \mathrm{~nm}$, length $0.5-1.5 \mu \mathrm{m}$ ) were obtained from Carbon Solutions, Inc. They were purified via thermal oxidation at $400{ }^{\circ} \mathrm{C}$ for $2 \mathrm{~h}$ in air, followed by refluxing in $6 \mathrm{M} \mathrm{HCl}$ solution overnight. Polycaprolactone (average $M_{\mathrm{w}} \sim 14000 \mathrm{Da}$ and average $M_{\mathrm{n}} \sim 10000 \mathrm{Da}$ ) as a control was purchased from Sigma-Aldrich. 3,3'-Dihydroxy-4,4'-diaminobiphenyl (HAB, 97\%) was purchased from Tokyo Chemical Industry and purified by recrystallization from an $N, N^{\prime}$-dimethylformamide (DMF)/water mixture. 4-Aminophenethyl alcohol (APA) was purified by recrystallization from ethanol. 2,2-Bis [4-(3,4-dicarboxyphenoxy)phenyl]propane anhydride (BPADA) was dissolved in acetic anhydride and toluene and refluxed; the solution was then cooled overnight and the solvent was removed under reduced pressure at $70{ }^{\circ} \mathrm{C}$. Epoxy resin (part A: EPR L20 and part B: EPH 960) was obtained from Bakelite. $N$-Methyl-2-pyrrolidone (NMP), $m$-xylene and $\varepsilon$-caprolactone (CL) were dried with calcium hydride and distilled before use. Other chemicals were used without purification.

\section{Synthesis of $\alpha, \omega$-dihydroxy-polyimide macroinitiator (OH-PI-OH)}

Under nitrogen flow, HAB (1.62 g, $7.50 \mathrm{mmol})$ and APA (0.69 g, $5.00 \mathrm{mmol}$ ) were dissolved in $15 \mathrm{~mL}$ of NMP with continuous stirring over $30 \mathrm{~min}$. Subsequently, BPADA (5.18 g, $10 \mathrm{mmol})$ was added into the solution. The reaction mixture was stirred at $25{ }^{\circ} \mathrm{C}$ for $10 \mathrm{~h}$, and then $10 \mathrm{~mL}$ of $m$-xylene was added into the poly(amic acid) solution. The solution was then refluxed at $180^{\circ} \mathrm{C}$ for $6 \mathrm{~h}$ to achieve thermal imidization (Scheme 1). The viscous product was slowly poured into a large amount of ethanol (volume ratio $=1: 10$ ), and the resulting precipitate was subsequently filtered. The $\mathrm{OH}-\mathrm{PI}-\mathrm{OH}$ product was dried at $70{ }^{\circ} \mathrm{C}$ for $24 \mathrm{~h}$ under reduced pressure. Other molecular weights of $\mathrm{OH}-\mathrm{PI}-\mathrm{OH}$ were synthesized by the same procedure as above with various ratios of the three monomers (Table 1). ${ }^{1} \mathrm{H}$ NMR (300 M, $d_{6}$-DMSO, $\left.25{ }^{\circ} \mathrm{C}\right): \delta 10.31(\mathrm{~s}, \mathrm{Ar}-\mathrm{OH})$, 7.88-8.04 (m, Ar-H), 7.40-7.21 (m, Ar-H), 7.21-7.02 (m, Ar- $H$ ), $4.76\left(\mathrm{~s},-\mathrm{CH}_{2}-\mathrm{OH}\right), 3.50$ (tr, $\left.-\mathrm{CH}_{2}-\mathrm{CH}_{2}-\right), 2.77\left(\mathrm{tr},-\mathrm{CH}_{2}-\mathrm{CH}_{2}-\right.$ ), $1.70\left(\mathrm{~s},-\mathrm{CH}_{3}\right)$.

\section{One-pot synthesis of triblock copolymer PCL-PI-PCL}

In a glove box, OH-PI-OH $(0.8 \mathrm{~g}, 0.1 \mathrm{mmol})\left(M_{\mathrm{n}}=12100 \mathrm{Da}\right)$ was first dissolved in $1 \mathrm{~mL} \mathrm{NMP}$, after which $\mathrm{Sn}(\mathrm{Oct})_{2}(0.081 \mathrm{~g}$, $0.2 \mathrm{mmol}$ ) in toluene was added into the solution. The mixture was stirred for $6 \mathrm{~h}$. Fresh distilled CL $(1.14 \mathrm{~g}, 10 \mathrm{mmol})$ was added into the mixture using a syringe. A highly viscous solution formed after $4 \mathrm{~h}$ and the polymerization was continued at $100{ }^{\circ} \mathrm{C}$ for another $24 \mathrm{~h}$. The crude product was poured into excess ethanol and the pale yellow precipitate was filtered. To obtain a pure triblock copolymer, the precipitate was dissolved in NMP and precipitated in methanol 2 times followed by drying overnight at $70{ }^{\circ} \mathrm{C}$ for $24 \mathrm{~h}$ under reduced pressure. Other PCL-PI-PCL triblock copolymers with different molecular weights (Table 2) were synthesized via a similar procedure.

\section{Dispersion of SWNTs with various dispersing agents in THF}

The concentration of SWNTs in solution can be estimated from the Beer-Lambert law, $A=\varepsilon l c$, where $A$ is the absorbance due to SWNTs at a particular wavelength (here we chose $700 \mathrm{~nm}), \varepsilon$ is the extinction coefficient, $l$ is the path length ( $1 \mathrm{~cm}$ for our cell), and $c$ is the concentration. To determine the extinction coefficient $(\varepsilon)$, the absorbance of dilute SWNT solutions $\left(0.025,0.05,0.075,0.125\right.$ and $0.2 \mathrm{mg} \mathrm{mL}^{-1}$ in THF with dispersing agent TB1a, prepared by tip sonication for 30 min ( 1 and 1 second on/off)) was measured and the absorbance at $700 \mathrm{~nm}$ was plotted against SWNT concentrations. The extinction coefficient was the slope of the absorbance/ SWNT concentration relationship.

$0.2 \mathrm{mg} \mathrm{mL}^{-1}$ solutions of TBs/SWNT (mass ratio of polymer and SWNTs $=2: 1)$ in THF were prepared by tip sonication ( $150 \mathrm{~W}, 60 \%$ ) for $30 \mathrm{~min}$ ( 1 and 1 second on/off) and the absorption at $700 \mathrm{~nm}$ was measured immediately and after centrifugation at $6000 \mathrm{rpm}$ for $10 \mathrm{~min}$ and $10000 \mathrm{rpm}$ for $10 \mathrm{~min}$. A $0.05 \mathrm{mg} \mathrm{mL}^{-1}$ solution of TB1a/SWNT (mass ratio of polymer and SWNTs $=2: 1$ ) in THF was prepared by tip sonication $(150 \mathrm{~W}, 60 \%)$ for $30 \mathrm{~min}$ ( 1 and 1 second on/off) and the absorption at $700 \mathrm{~nm}$ was measured immediately and after centrifugation at $6000 \mathrm{rpm}$ for $10 \mathrm{~min}$ and 10000 $\mathrm{rpm}$ for $10 \mathrm{~min}$. Comparison measurements were made of HP1/SWNT in THF at the same concentration and centrifuge preparation.

\section{Fabrication of TB1a/epoxy/SWNT films}

Epoxy films reinforced with SWNTs were fabricated by the solution casting method. L20 epoxy parts A and B were mixed and pre-cured at $40{ }^{\circ} \mathrm{C}$ for $30 \mathrm{~min}$. The dispersed TB1a/SWNTs $\left(1 \mathrm{mg} \mathrm{mL} \mathrm{m}^{-1}, 2 \mathrm{mg} \mathrm{mL}{ }^{-1}\right.$, etc.) in THF were prepared via tip sonication $(150 \mathrm{~W}, 60 \%)$ for $15 \mathrm{~min}$ and then added to the calculated amount of pre-cured epoxy. After thorough mixing 
by bath sonication, the solution was cast on glass. After evaporating the solvent, the films were fully dried in a vacuum oven at $40{ }^{\circ} \mathrm{C}$. The films were then peeled from the glass and cured at $130{ }^{\circ} \mathrm{C}$ for $3 \mathrm{~h}$.

\section{Structure analysis and characterization}

Molecular weights and molecular weight distributions were determined by gel permeation chromatography (GPC) performed with a Shimadzu LC-20A Series GPC system equipped with a pump, a BC-PL gel mixed column, and an RID-10A refractive index detector, using DMF with $0.02 \mathrm{M} \mathrm{LiBr}$ as the eluent and polystyrene standards as the reference. Raman characterization was carried out on a Renishaw Raman scope with a He-Ne laser at an excitation wavelength of $785 \mathrm{~nm}$. The dispersions were filtered through the PTFE membrane and dried in a vacuum to remove the solvents for Raman characterization. The absorption spectra of HP or TB-dispersed SWNT dispersions were recorded with a Varian Cary 5000 UV-vis-NIR spectrophotometer. ${ }^{1} \mathrm{H}$ NMR spectra were recorded at $25{ }^{\circ} \mathrm{C}$ using a Bruker AVANCE-300 (300 MHz) spectrometer with $\mathrm{CDCl}_{3}$ or $d_{6}$-DMSO as solvents and TMS as the internal reference. FTIR was performed with a Perkin Elmer Spectrum One at $4.0 \mathrm{~cm}^{-1}$ resolution. Atomic force microscopy (AFM) was conducted using a MFP 3D microscope in ac mode; PCL-PI-PCL/SWNT (mass ratio of polymer and SWNTs $=2: 1$ ) dispersion in THF was prepared via sonication and then deposited onto a clean silicon wafer by spin coating. High-resolution transmission electron microscopy (TEM) images were obtained using a JEOL Model 3010F analytical electron microscope operating at an accelerating voltage of $200 \mathrm{kV}$. TEM samples were prepared by placing several drops of HP1/SWNT (mass ratio of polymer and SWNTs $=2: 1$ ) suspension or TB1a/ SWNT (mass ratio of polymer and SWNTs $=2: 1$ ) dispersion in THF on a 200 mesh carbon-coated copper grid (Easylab) and drying at room temperature. Field-emission scanning electron microscopy (FE-SEM) images were acquired using a JEOL JSM-6700F microscope. The fractured surfaces of composite films were coated with a thin layer of gold prior to SEM observation. Tensile tests of composite films were performed on an Instron 5543 Tensile Meter with a $100 \mathrm{~N}$ load cell at ambient temperature. The crosshead speed was $2.5 \mathrm{~mm} \mathrm{~min}^{-1}$. Mechanical properties of each composite formulation were determined on five to ten specimens, and property averages and standard deviations were computed.

\section{Conclusion}

In summary, novel triblock copolymers of polycaprolactonepolyimide-polycaprolactone were successfully synthesized via a 2-step sequential polymerization process of polycondensation followed by ring-opening polymerization. This approach to triblock copolymer synthesis is versatile and efficient. The PCL-PI-PCL copolymer dispersed the SWNTs effectively in THF, even under high-speed centrifugation or upon $1 \mathrm{~h}$ standing. Among many potential applications, effective SWNT dis- persants can be applied in SWNT/epoxy composites to improve their mechanical properties. We showed that the optimized PCL-PI-PCL triblock-dispersed SWNTs (2 wt\%) led to a $74 \%$, $35 \%$ and $62 \%$ increase in the tensile strength, modulus and elongation of the nanotube/dispersant epoxy films compared to the control without nanotubes. Our triblock copolymers avoided the use of a high boiling solvent such as DMF for dispersing carbon nanotubes because of the good solubility of PCL blocks. The excellent nanotube dispersion properties of the copolymer were due to the combination of strong charge transfer between the nanotube and PI, as well as the good solubility of the PCL end blocks in the chosen solvent (THF). The replacement of caprolactone with other monomers may provide a route to the synthesis of highly effective SWNT dispersants in a wide range of solvents, which could in turn open up new ways to exploit SWNTs in widespread applications.

\section{Acknowledgements}

Liu Chengyin thanks Nanyang Technological University for the support of her Ph.D. We thank Dr Wang Yilei for his help in AFM measurements and Zhang Penghui for his help in TEM measurements. We also acknowledge the funding support from a Singapore Ministry of Education Tier 3 grant (MOE2013-T3-1-002), a Singapore Ministry of Health Industry Alignment Fund (NMRC/MOHIAFCAT2/003/2014), and the NTU iFood grant.

\section{References}

1 J. Che and M. B. Chan-Park, Adv. Funct. Mater., 2008, 18, 888-897.

2 W. Yuan, J. Che and M. B. Chan-Park, Chem. Mater., 2011, 23, 4149-4157.

3 W. Yuan, W. Li, Y. Mu and M. B. Chan-Park, ACS Appl. Mater. Interfaces, 2011, 3, 1702-1712.

4 J. Ju, Q. Wang, T. Wang and C. Wang, J. Colloid Interface Sci., 2013, 404, 36-41.

5 V. Delplace and J. Nicolas, Nat. Chem., 2015, 7, 771-784.

6 A. P. Filippov, E. V. Belyaeva, T. K. Meleshko and A. V. Yakimansky, J. Polym. Sci., Part B: Polym. Phys., 2014, 52, 1539-1546.

7 S. Miyata, K. Yoshida, H. Shirokura, M. Kashio and K. Nagai, Polym. Int., 2009, 58, 1148-1159.

8 S. Liu, L. Wei, L. Hao, N. Fang, M. W. Chang, R. Xu, Y. Yang and Y. Chen, ACS Nano, 2009, 3, 3891-3902.

9 J. Wang, J. Zhang, A. K. Sundramoorthy, P. Chen and M. B. Chan-Park, Nanoscale, 2014, 6, 4560-4565.

10 R. Martel, T. Schmidt, H. Shea, T. Hertel and P. Avouris, Appl. Phys. Lett., 1998, 73, 2447-2449.

11 K. Besteman, J.-O. Lee, F. G. Wiertz, H. A. Heering and C. Dekker, Nano Lett., 2003, 3, 727-730.

12 J. Wang, M. Musameh and Y. Lin, J. Am. Chem. Soc., 2003, 125, 2408-2409. 
13 Q. Cheng, M. Li, L. Jiang and Z. Tang, Adv. Mater., 2012, 24, 1838-1843.

14 J. Zhu, J. C. Kim, H. Peng, J. L. Margrave, V. N. Khabashesku and E. V. Barrera, Nano Lett., 2003, 3, 1107-1113.

15 J. Che, W. Yuan, G. Jiang, J. Dai, S. Y. Lim and M. B. ChanPark, Chem. Mater., 2009, 21, 1471-1479.

16 W. Yuan, J. Feng, Z. Judeh, J. Dai and M. B. Chan-Park, Chem. Mater., 2010, 22, 6542-6554.

17 M. X. Pulikkathara, O. V. Kuznetsov and V. N. Khabashesku, Chem. Mater., 2008, 20, 2685-2695.

18 Q. Yang, L. Shuai and X. Pan, Biomacromolecules, 2008, 9, 3422-3426.

19 K. T. Kim and W. H. Jo, J. Polym. Sci., Part A: Polym. Chem., 2010, 48, 4184-4191.

20 J. Zou, S. I. Khondaker, Q. Huo and L. Zhai, Adv. Funct. Mater., 2009, 19, 479-483.

21 H. Wang, J. Mei, P. Liu, K. Schmidt, G. Jiménez-Osés, S. Osuna, L. Fang, C. J. Tassone, A. P. Zoombelt and A. N. Sokolov, ACS Nano, 2013, 7, 2659-2668.
22 V. C. Moore, M. S. Strano, E. H. Haroz, R. H. Hauge, R. E. Smalley, J. Schmidt and Y. Talmon, Nano Lett., 2003, 3, 1379-1382.

23 S. Ando, Y. Koyama, S. Miyata, S. Sato, S. Kanehashi and K. Nagai, Polym. Int., 2014, 63, 1634-1642.

24 J. Zhu, J. Kim, H. Peng, J. L. Margrave, V. N. Khabashesku and E. V. Barrera, Nano Lett., 2003, 3, 1107-1113.

25 H. Q. Peng, L. B. Alemany, J. L. Margrave and V. N. Khabashesku, J. Am. Chem. Soc., 2003, 125, 1517415182.

26 A. M. Rao, P. C. Eklund, S. Bandow, A. Thess and R. E. Smalley, Nature, 1997, 388, 257-259.

27 S. Gayen, S. N. Behera and S. M. Bose, Phys. Rev. B: Condens. Matter, 2007, 76, 165433.

28 H. Kataura, Y. Maniwa, S. Masubuchi, S. Kazama, X. Zhao, Y. Ando, Y. Ohtsuka, S. Suzuki, Y. Achiba and R. Saito, AIP Conf. Proc., 2000, 262-265.

29 L. An, Y. Pan, X. Shen, H. Lu and Y. Yang, J. Mater. Chem., 2008, 18, 4928-4941. 